\title{
Field-aligned conductance values estimated from Maxwellian and kappa distributions in quiet and disturbed events using Freja electron data
}

\author{
A. Olsson ${ }^{1}$, P. Janhunen ${ }^{2,1}$ \\ ${ }^{1}$ Swedish Institute of Space Physics, Uppsala Division, Uppsala, Sweden \\ ${ }^{2}$ Finnish Meteorological Institute, Geophysical Research, Helsinki, Finland
}

Received: 12 February 1997 / Revised: 27 August 1997 / Accepted: 8 September 1997

\begin{abstract}
We study the question of what difference it makes for the derived field-aligned conductance $(K)$ values if one uses Maxwellian or kappa distributions for the fitting of low-orbiting satellite electron flux spectra in the auroral region. This question has arisen because sometimes a high-energy tail is seen in the spectra. In principle, the kappa fits should always be better, because the kappa distribution is a generalization of the Maxwellian. However, the physical meaning of the parameters appearing in the Maxwellian is clearer. It therefore makes sense to study under which circumstances it is appropriate to use a Maxwellian. We use Freja electron data (TESP and MATE) from two events. One of the events represents quiet magnetospheric conditions (stable arc) and the other represents disturbed conditions (surge). In these Freja events, at least, using kappa rather than Maxwellian fitting gives a better fit to the observed distribution, but the difference in $K$ values is not large (usually less than 20\%). The difference can be of either sign. However, sometimes even the kappa distribution does not provide a good fit, and one needs a more complicated distribution such as two Maxwellians. We investigate the relative contributions of the two Maxwellians to the total field-aligned conductance value in these cases. We find that the contribution of the highenergy population is insignificant (usually much less than $20 \%$ ). This is because $K$ is proportional to $n / \sqrt{E_{c}}$, where $n$ is the source plasma density and $E_{c}$ is the characteristic energy.
\end{abstract}

Key words. Field-aligned conductance $\cdot$ Maxwellian distribution - Kappa distribution - Freja electron data

\section{Introduction}

Electron flux energy spectra measured by satellites in the auroral region contain information of the source plasma properties (density $N_{e}$, characteristic energy $E_{c}$ ) and the acceleration region (the accelerating voltage $V$ ). In this paper we are mainly interested in the field-aligned conductance $K$ (Weimer et al., 1987) which depends on the source plasma properties. Traditionally, one has estimated these parameters by fitting an accelerated Maxwellian distribution to the data (Evans, 1974). In the Maxwellian case the field-aligned conductance is proportional to $N_{e} / \sqrt{E_{c}}$ (Lundin and Sandahl, 1978; Fridman and Lemaire, 1980).

However, sometimes one observes a high-energy tail in the electron flux energy spectra, which can not be fitted well by an accelerated Maxwellian (Vasyliunas, 1968; Sarris et al., 1981; Christon et al., 1988). These authors have used the kappa distribution (Vasyliunas, 1968) to describe these situations. The purpose of this paper is to investigate how sensitive the field-aligned conductance $K$ is to the choice of distribution function.

The accelerated Maxwellian particle flux is given by Evans (1974):

$F^{M}=\frac{1}{\pi} \frac{N_{e}}{\sqrt{2 \pi m_{e}}} \frac{E}{E_{c}^{3 / 2}} e^{-\left(\frac{E-e V}{E_{c}}\right)}$,

where $N_{e}$ is the source plasma density, $m_{e}$ is the electron mass, $E$ is the particle energy, $E_{c}$ is the characteristic energy (source plasma temperature in energy units) and $V$ is the accelerating potential. The accelerated kappa distribution particle flux is given by

$$
\begin{array}{r}
F^{\kappa}=\frac{1}{\pi} \frac{N_{e}}{\sqrt{2 \pi m_{e}}} \frac{E}{E_{c}^{3 / 2}} \frac{\Gamma(\kappa)}{\Gamma(\kappa-1 / 2) \sqrt{\kappa}} \\
{\left[1+\frac{E-e V}{\kappa E_{c}}\right]^{-\kappa-1},}
\end{array}
$$

(Lui and Krimigis, 1983), where $\Gamma$ is the Euler gamma function and $\kappa$ is the spectral index characterizing the distribution. For $\kappa \rightarrow \infty$ the kappa distribution 
approaches the Maxwellian distribution (Vasyliunas, 1968; Pierrard, 1996). The parameter $E_{c}$ (characteristic energy) is related to the thermal energy of the source plasma. Usually the source plasma temperature $E_{T}$ in energy units (thermal energy) is defined for any distribution function $f$ as

$E_{T}=\frac{2}{3} \frac{1}{N_{e}} \int f(\mathbf{v}) \frac{1}{2} m_{e} v^{2} d^{3} \mathbf{v}$

(Collier, 1995). Evaluating this integral for the kappa distribution yields

$E_{T}=\kappa E_{c} /(\kappa-3 / 2)$.

In the Maxwellian case it yields $E_{T}=E_{c}$.

In some cases not even the kappa distribution can provide a good fit to the high-energy tail. In these cases the tail consists rather of a different population such that the whole spectrum could be fitted by two Maxwellians. We will investigate these cases and see what the effect on the field-aligned conductances $K$ will be.

\section{Observations}

The Swedish-German satellite Freja was launched in 1992 (Lundin et al., 1994). With an inclination of 63, Freja often passes tangentially along the auroral oval at around $1750 \mathrm{~km}$ height, that is, most often below the mid-altitude auroral acceleration region (Lundin et al., 1994) and in the interaction zone between the magnetosphere and ionosphere.

In this study we use the two electron detectors of Freja. The TESP instrument (Boehm et al., 1994) measures in the low energy range from $20 \mathrm{eV}$ to 30 $\mathrm{keV}$. The MATE instrument (Eliasson et al., 1994) measures in the high energy range from $1 \mathrm{keV}$ to 100 $\mathrm{keV}$. TESP has 32 and MATE has 16 energy channels.

We have selected two events for this study, which we will refer to using the Freja orbit numbers. Event 869 is a surge event; event 907 is a quiet arc event. Since these events have been studied in Olsson et al. (1997a), we will not discuss the geophysical context any further in the present paper. On the surge head of event 869 the energies were so high that the MATE instrument had to be used. Otherwise, TESP data were used in both events.

The fitting procedure is as follows. We first determine the accelerating potential $V$ from the peak position in the particle flux (see Fig. 1 for an example). The integration time was around $0.5 \mathrm{~s}$ for both TESP and MATE data. The most field-aligned direction among the sweeped directions was used. All data points lower than the peak are then removed. We use the LevenbergMarquardt method (Press et al., 1992) for fitting. In the case of Maxwellian distribution the number of fitted variables is three $\left(N_{e}, V, E_{c}\right)$. For kappa distribution there is also the fourth parameter $\kappa$ to be fitted. The fitting routine seems to work better if $V$ is included in the list of varied parameters rather than keeping it completely fixed to the peak position, although the variation of $V$ during the fitting process is small. Different initial values were tried manually and all fits

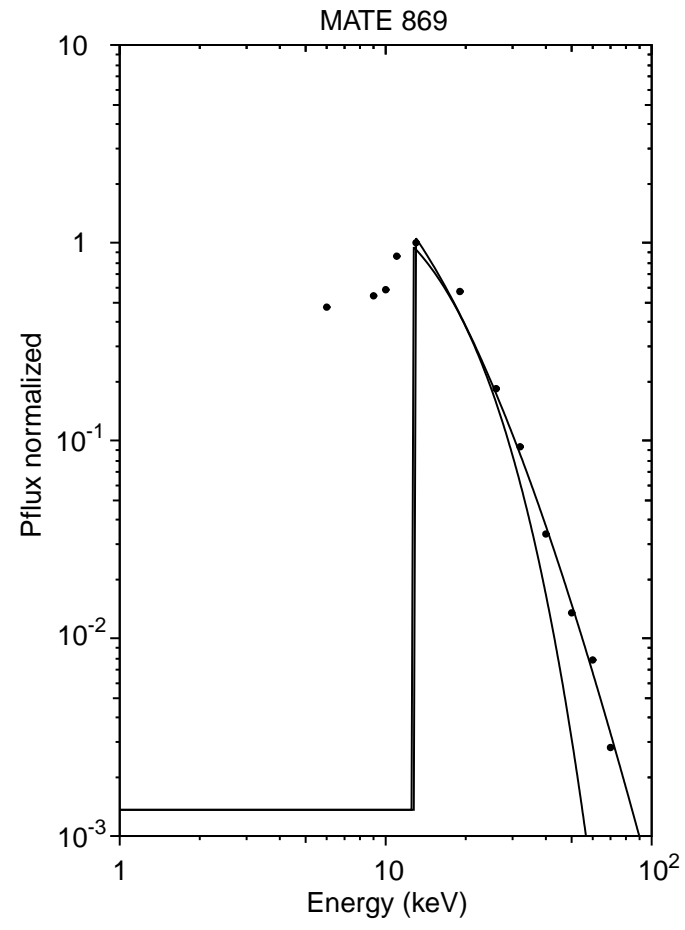

Fig. 1. Example of kappa and Maxwellian fits in a case where the kappa fit seems to be better. The characteristic energy $E_{c}$ is $5.04 \mathrm{keV}$ for the kappa and $5.25 \mathrm{keV}$ for the Maxwellian. The accelerating voltage is $13 \mathrm{keV}$. The kappa value is 6.1 . The source densities are 0.069 and $0.065 \mathrm{~cm}^{-3}$ for the kappa and Maxwellian fits, respectively. The corresponding $K$ values are $2.41 \cdot 10^{-11}$ and $2.46 \cdot 10^{-11}$ for the Maxwellian and for the kappa distribution, respectively

were manually checked and any suspicious cases were excluded. The counts of succeeded and failed fits are summarized in Table 1.

In cases where the fits did not succeed there was either a pronounced high-energy tail that would have required a double Maxwellian function (with six parameters) or the peak position could not be determined. The latter case may happen either because there is no accelerating potential at that point or because there is some superposed diffuse precipitation that masks the peak. The fact that a substantial fraction of the fits are rejected is not a problem in this paper, because our aim is a statistical study of the difference between the two fitting methods.

In Fig. 1 we show a typical case where the kappa distribution provides a better fit than the Maxwellian for MATE data. The corresponding parameters are provid-

Table 1. Number of successful and failed fits in each event. Fit was said to succeed if both Maxwellian and Kappa fits produced an acceptable result (apart from the fact that the Maxwellian fit might miss the tail). (The numbers in parentheses correspond to cases when at least one of the fits succeeded.)

\begin{tabular}{lcll}
\hline & failed fits & successful fits & total \\
\hline mate869 & 91 & 72 & 163 \\
tesp869 & $311(268)$ & $35(78)$ & 346 \\
tesp907 & $138(132)$ & $66(72)$ & 204 \\
\hline
\end{tabular}


ed in the figure caption. The $K$ values were almost equal in this example, although the Maxwellian fit seems to be rather bad visually.

As we could not find any marked trends concerning the differences between $K^{\kappa}$ and $K^{M}$ in our two events, we plot the $K^{\kappa} / K^{M}$ ratio for all data points from the events including both TESP and MATE data in Fig. 2. The difference between $K^{\kappa}$ and $K^{M}$ is usually less than $20 \%$. The solid and dashed curves in Fig. 2 are explained in Sect. 3.

In Fig. 3 we show two examples where it was not possible to carry out a proper kappa fitting to the highenergy tail, because the tail seems to be a separate (Maxwellian or kappa) population. Figure. 3a represents an extreme case where the higher-energy population is strong. We fit two accelerated Maxwellians in Fig. 3a. The problem with the fitting is that the position

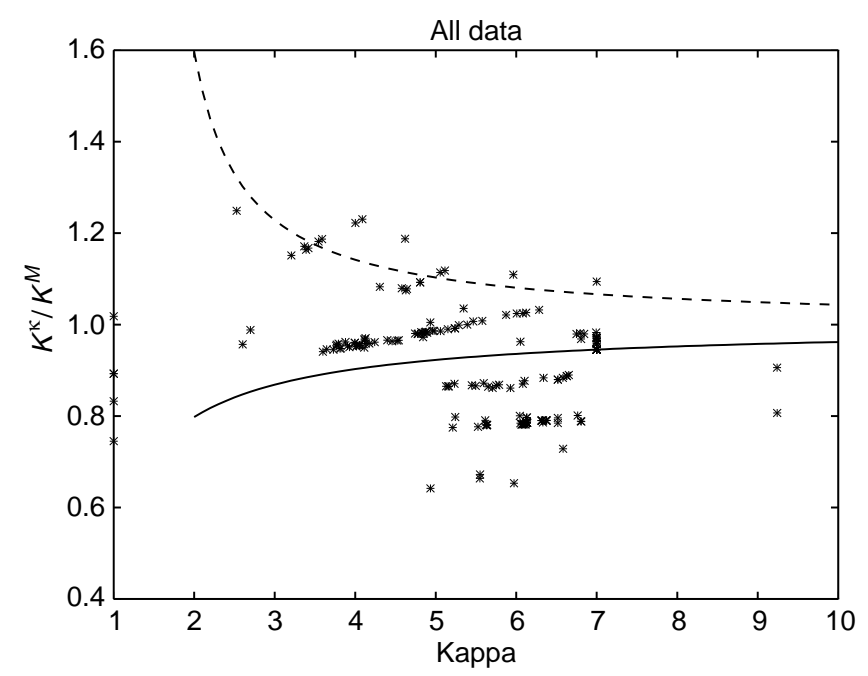

Fig. 2. The ratio between $K^{\kappa}$ and $K^{M}$ for all successfully fitted data points as a function of the fitted $\kappa$. The solid curve is Eq. (10) and the dashed curve Eq. (11). The difference between $K^{\kappa}$ and $K^{M}$ is usually less than $20 \%$ of the peak of the higher-energy population is not clear. If one assumes that the higher-energy population starts where there is the kink in the spectrum, i.e. at around 6 $\mathrm{keV}$, then the contribution of the higher-energy population to the total $K$ value is only about $5 \%$. However, it is much more probable that the higher-energy population has been accelerated through the same potential drop $V$ as the lower-energy population. Thus we must extrapolate the higher-energy curve into the energy range (about $4-6 \mathrm{keV}$ ) which is masked by the lowerenergy population peak. This would give a contribution to the total $K$ value of less than $20 \%$. A corresponding kappa fit to the higher-energy population would contribute somewhat less. We tried to carry out the extrapolation, which is inherently ambiguous, in such a way as to maximize the contribution of the higherenergy population to the $K$ value. Thus the value $20 \%$ can be considered as an upper limit in this example.

In Fig. $3 b$ we show a more typical case of the highenergy tail. In a spectrum like this it is not possible to fit a single Maxwellian or kappa distribution to the whole spectrum, and one has rather to use two Maxwellians (or kappa distributions) as was done in Fig. 3a. In this case we expect an even lower contribution to the total $K$ value than found in Fig. 3a.

\section{Discussion}

In Fig. 1 we saw a typical example of a case where the Maxwellian function fit was visually poor (at least much worse than the kappa fit), but the derived field-aligned conductance values were nevertheless almost equal. If the distribution function is a superposition of two or more distributions, the total $K$ value can be obtained by adding the component $K$ values together (Olsson and Janhunen, 1997b) and in Fig. 3a we saw that the highenergy tail contributes only little to the total $K$ value. A physical explanation as to why the high-energy tail population contribution is small is that the $K$ parameter
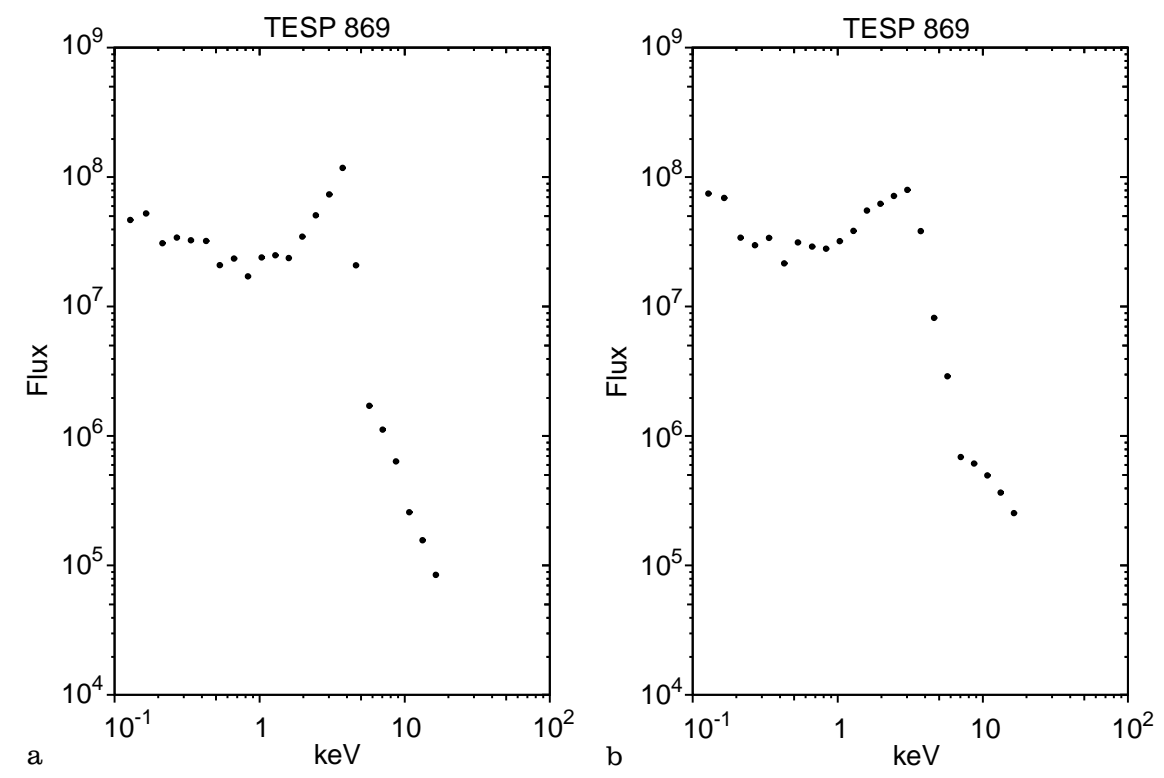

Fig. 3. a Example of an extreme case where the distribution has a significant tail that cannot be fitted well by either a single Maxwellian or a kappa distribution. Using two Maxwellians gives a good fit. The contribution of the higher-energy population to the total $K$ value is less than 20\%, however. b Example of a more typical case where the distribution again has a tail that could be fitted using a double Maxwellian but the relative contribution of the tail to the total $K$ value would be smaller than in part a of the figure 
is proportional to $N_{e} / \sqrt{E_{c}}$ for both Maxwellian and kappa distributions. For the high-energy tail both $N_{e}$ is smaller and $E_{c}$ is larger. This explanation applies only to the case where we have two superposed (Maxwellian or kappa) populations. A more general explanation is as follows. Using the general formulas given by Janhunen and Olsson (1997), it is easy to show that the $K$ parameter for any distribution function $f$ is given by

$K=\frac{2 \pi e^{2}}{m^{2}} \int_{0}^{\infty} f(E) d E$

if the magnetic field in the source plasma region is very small compared to magnetic field at Freja altitude (1750 $\mathrm{km}$ ), as is almost always the case. [Notice, however, that the field-aligned current is given by $j=K V$ only if the additional assumption $\mathrm{eV} \gg E_{c}$ is valid as well (Lundin and Sandahl, 1978; Fridman and Lemaire, 1980).] Thus the relative contribution of the high-energy tail is small because the distribution function $f$ is small there. Here the distribution function $f(E)$ is given by

$f^{M}(E)=N_{e}\left(\frac{m_{e}}{2 \pi E_{c}}\right)^{3 / 2} e^{-E / E_{c}}$

in the Maxwellian case and by

$f^{\kappa}(E)=\frac{N_{e} \Gamma(\kappa)}{\Gamma(\kappa-1 / 2) \sqrt{\kappa}}\left(\frac{m_{e}}{2 \pi E_{c}}\right)^{3 / 2}\left(1+\frac{E}{\kappa E_{c}}\right)^{-\kappa-1}$

in the kappa distribution case. These formulas, together with the information of how they are normalized, can be found in Janhunen and Olsson (1997), but we also give them here to make notation clear. Notice that the quantities $F^{M}$ and $F^{\kappa}$ defined by eqs. (1) and (2) in the present paper are particle fluxes that are closely related but not equal to the distribution functions $f^{M}$ and $f^{\kappa}$ defined by Eqs. (6) and (7).

The field-aligned conductance in the case of Maxwellian distribution $K^{M}$ is given by

$K^{M}=\frac{e^{2} N_{e}^{M}}{\sqrt{2 \pi m_{e} E_{c}^{M}}}$

(Lundin and Sandahl, 1978,Fridman and Lemaire, 1980]. The $K$ parameter in the kappa distribution case is

$K^{\kappa}=\frac{e^{2} N_{e}^{\kappa}}{\sqrt{2 \pi m_{e} E_{c}^{\kappa}}} \frac{\Gamma(\kappa+1)}{\Gamma(\kappa-1 / 2) \kappa^{3 / 2}}$

(Janhunen and Olsson, 1997). In these formulas $N_{e}^{M}$ and $N_{e}^{\kappa}$ are the electron densities resulting from Maxwellian and kappa fitting, respectively, and similarly for the characteristic energies $\left(E_{c}^{M}, E_{c}^{\kappa}\right)$. Equations (8) and (9) can also be derived directly using the general formula in Eq. (5) as well as Eqs. (6) and (7) . For simplicity we will assume that the source plasma densities $N_{e}^{M}$ and $N_{e}^{\kappa}$ are exactly the same. Thus the $K$ parameter ratio will be

$\frac{K^{\kappa}}{K^{M}}=\sqrt{\frac{E_{c}^{M}}{E_{c}^{\kappa}}} \frac{\Gamma(\kappa)}{\Gamma(\kappa-1 / 2) \sqrt{\kappa}}$.
The lower (solid) curve in Fig. 2 has been produced by assuming that $E_{c}^{\kappa}=E_{c}^{M}$ in Eq. (10), i.e. that the characteristic energies are equal. In this case Eq. (10) is always smaller than unity. The same curve can be found in Fig. 5 of Janhunen and Olsson (1997). In the case of kappa distribution, however, the characteristic energy $E_{c}^{\kappa}$ is not the same as the thermal energy $E_{T}^{\kappa}$, but is related to it by Eq. (4). Therefore, we have generated the upper (dashed) curve in Fig. 2 by assuming that the thermal energies (true temperatures) of the source plasmas are equal, that is $E_{T}^{M}=E_{T}^{\kappa}$ (where $E_{T}^{M}=E_{c}^{M}$, as already mentioned). Thus, in this case the $K$ ratio assumes the form

$\frac{K^{\kappa}}{K^{M}}=\sqrt{\frac{\kappa}{\kappa-3 / 2}} \frac{\Gamma(\kappa)}{\Gamma(\kappa-1 / 2) \sqrt{\kappa}}$,

which is always larger than unity.

Since the kappa distribution is a generalization of the Maxwellian, in cases where the Maxwellian and kappa fits produce different $K$ values, $K^{\kappa}$ should always be a better estimate of the true $K$ value than $K^{M}$. Thus the ratio $K^{\kappa} / K^{M}$ can be larger or smaller than unity and neither of Eqs. (10) or (11) usually holds because these are based on idealized assumptions of equal characteristic and thermal energies, respectively. However, when $\kappa \rightarrow \infty$, both Eqs. (10) and (11) approach unity, because then the kappa distribution becomes identical to the Maxwellian.

Thus the two "theoretical" curves appearing in Fig. 2 together with the data points are not any strict limits that the data points should obey. However, their deviation from unity should be of the same order of magnitude as the deviation of the data points from unity, and this indeed seems to be the case.

\section{Summary}

We summarize our findings briefly.

1. The field-aligned conductance $K$ derived using kappa fitting differs from the Maxwellian-fitting result usually by less than $20 \%$ in both quiet and disturbed events. An analytic explanation is provided by Eq. (5).

2. The theory cannot predict whether the ratio $K^{\kappa} / K^{M}$ is larger or smaller than unity because for both Maxwellian and kappa fits to be good simultaneously the kappa value must be large, in which case the ratio is anyway very close to unity. This is in accordance with our data (Fig. 2), where the $K^{\kappa} / K^{M}$ ratio fluctuates around unity.

3. As far as one is interested in determining the fieldaligned conductance $K$, using single Maxwellian fitting gives almost as good a result as kappa fitting.

4. In rather many cases the kappa fit also fails and one is forced to use two Maxwellians. However, even in these cases the contribution of the hotter population to the $K$ value is less than $20 \%$. Thus one can obtain almost the correct $K$ value by fitting a single Maxwellian to the low-energy part and ignoring the higher-energy population. 
5. A physical explanation as to why the high-energy population contributes little to the $K$ value is that $K$ is proportional to $N_{e} / \sqrt{E_{c}}$. $K$ value contributions from different source plasma distributions are additive.

6. The kappa values fluctuate between 4 and 7 in a seemingly random fashion usually during the events. No clear differences between the two events could be seen in this regard, so no conclusions concerning, e.g., the frequency of low kappa distributions in various geomagnetic activity levels could be drawn from our data.

Acknowledgements. The Freja project was supported by the Swedish National Space Board (SNSB) and by the Deutsche Agentur für Raumfahrtangelegenheiten (DARA). The Freja satellite is managed and operated by the Swedish Space Corporation. We especially thank L. Andersson from the Kiruna group for providing the $\mathrm{F} 3 \mathrm{H} / \mathrm{MATE}$ data and $\mathrm{J}$. Clemmons and the $\mathrm{F} 7$ team for the TESP data.

Topical Editor D. Alcaydé thanks T. Iijima and another referee for their help in evaluating this paper.

\section{References}

Boehm, M., G. Paschmann, J. Clemmons, H. Höfner, R. Frenzel, M. Ertl, G. Haerendel, P. Hill, H. Lauche, L. Eliasson, and R. Lundin, The TESP electron spectrometer and correlator (F7) on Freja. Space Sci. Rev., 70, 509-540, 1994.

Christon, S. P., D. G. Mitchell, D. J. Williams, L. A. Frank, C. Y. Huang, and T. E. Eastman, Energy spectra of plasma sheet ions from $\sim 50 \mathrm{eV} / \mathrm{e}$ to $\sim 1 \mathrm{MeV}$ during plasma temperature transitions, J. Geophys. Res., 93, 2562-2572, 1988.

Collier, M. R., The adiabatic transport of superthermal distributions modelled by kappa functions, Geophys. Res. Lett., 22, 2673-2676, 1995.

Eliasson, L., O. Norberg, R. Lundin, K. Lundin, S. Olsen, H. Borg, M. André, H. Koskinen, P. Riihelä, M. Boehm, and B. Whalen, The Freja hot plasma experiment - instrument and first results, Space Sci. Rev., 70, 563-576, 1994.
Evans, D.S., Precipitating electron fluxes formed by a magnetic field-aligned potential difference, J. Geophys. Res., 79, 28532858, 1974.

Fridman, M., and J. Lemaire, Relationship between auroral electron fluxes and field-aligned electric potential differences, J. Geophys. Res., 85, 664-670, 1980.

Janhunen, P., and A. Olsson, The current-voltage relationship revisited: exact and approximate formulas with almost general validity for hot magnetospheric electrons for bi-Maxwellian and kappa distributions, Ann. Geophysicae, this issue, 1997.

Lui, A. T. Y., and S. M. Krimigis, Energetic ion beam in the Earth's magnetotail lobe, Geophys. Res. Lett., 10, 13-16, 1983.

Lundin, R., and I. Sandahl, Some characteristics of the parallel electric field acceleration of electrons over discrete auroral arcs as observed from two rocket flights, ESA SP-135, 125-136, 1978.

Lundin, R., G. Haerendel, and S. Grahn, The Freja science mission, Space Sci. Rev., 70, 405-419, 1994.

Olsson, A., L. Andersson, A. I. Eriksson, J. H. Clemmons, R. E. Erlandson, G. Reeves, T. Hughes, and J. S. Murphree, Freja studies of the current-voltage relation in substorm related events, J. Geophys. Res., submitted, 1997a.

Olsson, A., and P. Janhunen, A case study of electron precipitation in the late growth phase on and nearby a preonset arc, Ann. Geophysicae, submitted, 1997b.

Pierrard, V., New model of magnetospheric current-voltage relationship, J. Geophys. Res., 101, 2669-2675, 1996.

Press, W. H., S. A. Teukolsky, W. T. Vetterling, and B. P. Flannery, Numerical Recipes in $C$, the art of scientific computing, 2nd edn., Cambridge, 1992.

Sarris, E. T., S. M. Krimigis, A. T. Y. Lui, K. L. Ackerson, L. A. Frank, and D. J. Williams, Relationship between energetic particles and plasmas in the distant plasma sheet, Geophys. Res. Lett., 8, 349-352, 1981.

Vasyliunas, V. M., A survey of low-energy electrons in the evening sector of the magnetosphere with OGO1 and OGO3, J. Geophys. Res., 73, 2839-2884, 1968.

Weimer, D. R., D. A. Gurnett, C. K. Goertz, J. D. Menietti, J. L. Burch, and M. Sugiura, The current-voltage relationship in auroral current sheets, J. Geophys. Res., 92, 187-194, 1987. 